\title{
Herpes zoster in patients with systemic lupus erythematosus: Clinical features, complications and risk factors
}

\author{
DONGYING CHEN ${ }^{1}$, HAO LI $^{1}$, JINGYI XIE ${ }^{2}$, ZHONGPING ZHAN $^{1}$, LIUQIN LIANG $^{1}$ and XIUYAN YANG ${ }^{1}$ \\ ${ }^{1}$ Department of Rheumatology, The First Affiliated Hospital of Sun Yat-sen University, Guangzhou, Guangdong 510080; \\ ${ }^{2}$ Department of Rheumatology, Shenzhen People's Hospital, Shenzhen, Guangdong 518020, P.R. China
}

Received January 5, 2016; Accepted September 7, 2017

DOI: $10.3892 /$ etm.2017.5297

\begin{abstract}
Herpes Zoster (HZ) is reported as the most prevalent viral infection in patients with systemic lupus erythematosus (SLE). The aim of the present study was to investigate the clinical features, complications, and potential risk factors of $\mathrm{HZ}$ in patients with SLE from Southern China. A retrospective study was performed among patients with SLE admitted to the First Affiliated Hospital of Sun Yet-Sen University (Guangzhou, China) between 2009 and 2013. Demographic information, clinical and laboratory data, and medications used were collected and analyzed. A total of 48 instances of $\mathrm{HZ}$ from 46 individuals in a cohort of 1,265 SLE patients during the follow-up period were identified, with an overall prevalence of $3.6 \%$. Complications occurred in $23.9 \%$ of patients with HZ (11/46). The risk of HZ was highest within 3-6 months following SLE diagnosis and reduced thereafter. The multivariate logistic regression analysis demonstrated that lymphopenia $(\mathrm{OR}=4.6$; 95\% $\mathrm{CI}=1.5-13.8 ; \mathrm{P}=0.006)$ and treatment with high-dose glucocorticoids $(\mathrm{GC} ; \mathrm{OR}=4.3 ; 95 \% \mathrm{CI}=1.6-11.7 ; \mathrm{P}=0.004)$ were both significantly associated with occurrence of $\mathrm{HZ}$. Lymphopenia was the only independent risk factor for the occurrence of complicated $\mathrm{HZ}(\mathrm{OR}=15.2$; 95\% $\mathrm{CI}=2.7-85.1$; $\mathrm{P}=0.002)$. There are some notable characteristics of $\mathrm{HZ}$ in patients with SLE in Southern China, such as the tendency to manifest in an early stage of SLE, and frequent complications with benign outcomes. The present data supported the role of lymphopenia and high-dose of GC therapy as risk factors for the occurrence of HZ. Lymphopenia was also shown to contribute to complicated HZ.
\end{abstract}

Correspondence to: Dr Zhongping Zhan, Department of Rheumatology, The First Affiliated Hospital of Sun Yat-sen University, 58 Zhongshan 2nd Road, Guangzhou, Guangdong 510080, P.R. China

E-mail: zhanchuyue@163.com

Key words: herpes zoster, systemic lupus erythematosus, complication, lymphopenia

\section{Introduction}

Herpes Zoster (HZ), which is a form of recurrent infection of varicella zoster virus (VZV), typically occurs decades after an instance of childhood VZV attack (1). It occurs most commonly in otherwise healthy, elderly individuals and immunocompromised hosts, including those with acquired immune deficiency syndrome, malignancies and systemic lupus erythematosus (SLE), amongst other conditions (2). The annual incidence of $\mathrm{HZ}$ is $1.5-3.0$ per 1,000 persons in the general population in the United States and markedly increased to 6.4-32.5 per 1,000 person-years in patients with SLE (3-6). Furthermore, compared with healthy individuals, patients with SLE and HZ have a greater tendency to develop cutaneous and visceral dissemination of lesions (7), which are significantly associated with a poor prognosis $(7,8)$. Furthermore, those with $\mathrm{HZ}$ are also more likely to develop bacterial superinfection during their disease course (9). At present, the most effective way to reduce the incidence and severity of $\mathrm{HZ}$ is zoster vaccination $(10,11)$. Despite the overall efficacy of the zoster vaccine, this is uncertain in SLE patients and has a high financial cost $(12,13)$. The zoster vaccine is currently not available in China, therefore patients who require treatment have to go abroad. Consequently, it is essential for clinicians to identify the risk factors that may predispose patients with SLE to the development of HZ. Risk factors predisposing SLE patients to $\mathrm{HZ}$ infection are not well established. A number of previous studies have attempted to identify risk factors for $\mathrm{HZ}$ in SLE; however, the findings of these studies were inconsistent $(9,14,15)$. Furthermore, whereas the role of leucopenia has been extensively analyzed (15), lymphopenia as a risk factor for $\mathrm{HZ}$ is seldom discussed. However, incidence rates, patient history and risk factors differ markedly by geographical location and among different ethnic groups (3-6), possibly due to genetic and environmental factors. Little is known about the clinical features of SLE when complicated with $\mathrm{HZ}$ in Southern China.

It was hypothesized that a study of $\mathrm{HZ}$ in patients with SLE in Southern China may help increase the awareness of the extent and natural history of this disease in the region, assess therapeutic strategies on higher risk patients with $\mathrm{HZ}$, and identify any subgroup(s) of patients who may benefit the most from the new zoster vaccine. In these regards, a retrospective study was performed to systematically examine the 
prevalence, nature and complications of $\mathrm{HZ}$ in patients with SLE in a single rheumatology center. Clinical and laboratory features, as well as administered therapies were reviewed to determine their association with the occurrence of $\mathrm{HZ}$ as well as the complications of $\mathrm{HZ}$.

\section{Patients and methods}

Study design and patients. A retrospective study was conducted using clinical records from the First Affiliated Hospital of Sun Yat-Sen University (Guangzhou, China) from January 2009-January 2013. A total of 1,265 patients recruited from the rheumatology outpatient clinics and inpatients department were diagnosed with SLE and fulfilled $\geq 4$ of the American College of Rheumatology revised classification criteria for SLE (1997) (16). A diagnosis of HZ was clinically established by the presence of a typical vesicular eruption developing in a dermatomal distribution (14). Viral isolation by culture or serology was not done for confirmation of HZ. For the present study, only those patients with a history of $\mathrm{HZ}$ following the diagnosis of SLE were included. Patients with a history of HZ onset prior to SLE were excluded. Among these SLE patients, 46 were diagnosed with HZ, and another 48 age- and gender-matched SLE patients without history of HZ were randomly selected as control.

Review of the clinical files of these 94 SLE patients was performed and data was extracted. From the initial diagnosis of SLE, patients were subsequently followed-up until the occurrence of HZ. Follow-up also ended in instances of death or if there was no episode of $\mathrm{HZ}$ prior to the last documented visit.

Disease definitions. Complications of $\mathrm{HZ}$ were defined as the occurrence of one or more of the following conditions: i) Ocular, visceral or neurological involvements caused by VZV (17); ii) chronic (lasting for $>30$ days) atypical skin lesions; iii) postherpetic neuralgia (defined as pain persisting for $>6$ weeks following initial appearance of the rash) (8); iv) cutaneous dissemination (defined as vesicular lesions outside the primary and adjacent dermatomes) (7); and v) infections associated with $\mathrm{HZ}$ that required treatment with antibiotics.

Leucopenia was defined as a total white blood cell count $<4.0 \times 10^{9} / 1$. Neutropenia was defined as a total neutrophil count $<1.5 \times 10^{9} / 1$. Lymphopenia was defined as a total lymphocyte count $<1.0 \times 10^{9} / 1$. Monocytosis was defined as a total monocyte count $>1.0 \times 10^{9} / 1$.

Demographic, clinical, laboratory data and therapeutic variables. The following data were collected from medical records: Demographic information including gender and age at SLE diagnosis; duration between the onset of SLE and $\mathrm{HZ}$; lupus disease activity; complications of HZ; clinical symptoms and signs; and laboratory findings such as blood routine test, various autoantibodies, erythrocyte sedimentation rate and $\mathrm{C}$-reactive protein. The use of therapeutic agents including glucocorticoids (GC) and other immunosuppressive agents (ISA) within 1 month of $\mathrm{HZ}$ onset was also recorded. The dosage of GC was defined as none, low-dose $(<30 \mathrm{mg}$ prednisone or equivalent per day) or high-dose $(\geq 30 \mathrm{mg}$ prednisone or equivalent per day). The SLE Disease Activity Index (SLEDAI) was used to evaluate SLE activity during HZ, and patients were defined as active SLE if the SLEDAI score was $\geq 6$ (18).

Statistical analysis. Data was analyzed using SPSS 16.0 (SPSS, Inc., Chicago, IL, USA). Continuous data are presented as the mean \pm standard deviation. Categorical variables were presented as the absolute value and percentage. The Mann-Whitney $U$ test was used to compare differences in continuous variables, and the chi-squared test was used for categorical variables. Variables that demonstrated significant associations with dependent variables in univariate analysis were included in a stepwise multivariate logistic regression analysis. Odds ratios (ORs) and 95\% confidence intervals (CIs) were also calculated. $\mathrm{P}<0.05$ was considered to indicate a statistically significant difference.

\section{Results}

Demographic data. In the present retrospective analysis, from a total of 1,265 patients with SLE, 48 instances of HZ were recorded in 46 patients. In total, $\mathrm{HZ}$ patients accounted for a prevalence of $3.6 \%$. Of these 48 patients, 2 experienced multiple episodes ( 2 each) of $\mathrm{HZ}$, whereas the remaining 44 patients experienced only one episode during the follow-up period.

All subjects were of Chinese Han ethnicity. As presented in Table I, the majority of patients with $\mathrm{HZ}$ were female $(95.7 \%)$

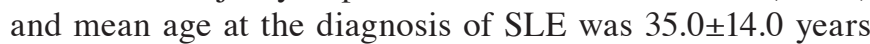
(range, 18-75 years). The mean duration between the diagnosis of SLE and HZ was 9.1 \pm 1.3 months, with a range from 0.5-36.0 months. Following SLE diagnosis, the risk of HZ was highest within 3-6 months and reduced thereafter (Fig. 1). The majority $(31 / 46 ; 67.4 \%)$ of patients experienced $\mathrm{HZ}$ in this period, whereas the remaining 15 patients developed $\mathrm{HZ}$ in the chronic stage of SLE ( $>6$ months).

Clinical characteristics of SLE patients with HZ. HZ skin lesions were most commonly located in the thoracic region $(37 / 48 ; 77.1 \%)$, followed by the lumbar segment $(6 / 48 ; 12.5 \%)$ and sacral area $(3 / 48 ; 6.3 \%)$. The dermatome occurred in trigeminal regions in 2 instances $(2 / 48,4.2 \%), 1$ in the inner corner of the eyelids and 1 on the tip of the ear.

A total of 35 patients exhibited typical skin lesions without complications. Of the remaining 11 patients who had complications in their disease course, $4(8.7 \%)$ had superimposed infections that required antibiotic therapy ( 2 with pneumonia, 2 with sepsis and 1 with cellulitis), and 7 (15.2\%) had postherpetic neuralgia. Only 2 of these 7 patients with postherpetic neuralgia had a course longer than 12 months. No patients in the present cohort experienced other serious complications, such as ocular, visceral or neurological involvement, chronic atypical skin lesions, and cutaneous dissemination.

Outcome and treatment. All patients received antiviral treatment with oral acyclovir or ganciclovir. A total of 10 patients $(21.7 \%)$ were ultimately treated with intravenous ganciclovir due to clinical resistance to acyclovir. All cases had complete resolution of rash with no cutaneous scarring. There were no deaths attributed to $\mathrm{HZ}$ in the present cohort. 
Table I. Comparison between SLE patients with and without HZ.

\begin{tabular}{|c|c|c|c|}
\hline Characteristic & With HZ $(n=46)$ & Without HZ (n=48) & P-value \\
\hline \multicolumn{4}{|l|}{ Demographic characteristics } \\
\hline Sex, male:female & $2: 44$ & $7: 41$ & 0.2 \\
\hline Age at SLE diagnosis, years & $35.0 \pm 14.0$ & $33.8 \pm 14.4$ & 0.7 \\
\hline \multicolumn{4}{|l|}{ Laboratory data } \\
\hline ANA positive, n (\%) & $45(97.8)$ & $43(89.6)$ & 0.2 \\
\hline Anti-dsDNA positive, n (\%) & $27(58.7)$ & $24(50.0)$ & 0.4 \\
\hline Anti-Sm antibody positive, n (\%) & $3(6.5)$ & $8(16.7)$ & 0.2 \\
\hline ACL positive, n (\%) & $3(6.5)$ & $9(18.8)$ & 0.1 \\
\hline Leucopenia, n (\%) & $7(15.2)$ & $12(25.0)$ & 0.3 \\
\hline Neutropenia, n (\%) & $6(13.0)$ & $8(16.7)$ & 0.8 \\
\hline Lymphopenia, n (\%) & $17(37.0)$ & $6(12.5)$ & 0.008 \\
\hline Monocytosis, n (\%) & $18(39.1)$ & $14(29.2)$ & 0.4 \\
\hline Anemia, n $(\%)$ & $29(63.0)$ & $24(50.0)$ & 0.2 \\
\hline Hypoproteinemia, n (\%) & $26(56.5)$ & $23(47.9)$ & 0.4 \\
\hline $\mathrm{ESR}, \mathrm{mm} / \mathrm{h}$ & $36.8 \pm 30.7$ & $44.1 \pm 23.9$ & 0.2 \\
\hline CRP, mg/l & $24.3 \pm 61.7$ & $6.7 \pm 12.0$ & 0.08 \\
\hline \multicolumn{4}{|l|}{ Clinical features } \\
\hline Renal involvement, n (\%) & $36(78.3)$ & $27(56.2)$ & 0.03 \\
\hline Neuro-psychiatric manifestations, n (\%) & $3(6.5)$ & $3(6.2)$ & 1 \\
\hline Articular, n (\%) & $20(43.5)$ & $23(47.9)$ & 0.7 \\
\hline Mucocutaneous involvement, n (\%) & $21(45.7)$ & $26(51.0)$ & 0.6 \\
\hline Serositis, $\mathrm{n}(\%)$ & $5(10.9)$ & $7(14.6)$ & 0.7 \\
\hline SLEDAI & $9.1 \pm 7.4$ & $5.4 \pm 4.2$ & 0.004 \\
\hline Active lupus, n (\%) & $25(54.3)$ & $14(29.2)$ & 0.02 \\
\hline Number of organs involved & $2.4 \pm 1.3$ & $2.3 \pm 1.2$ & 0.6 \\
\hline \multicolumn{4}{|l|}{ Treatments prior to $\mathrm{HZ}$ onset } \\
\hline Cyclophosphamide, n (\%) & $20(43.5)$ & $17(35.4)$ & 0.4 \\
\hline Mycophenolate motifile, n (\%) & $8(17.4)$ & $9(18.8)$ & 0.9 \\
\hline Methotrexate, n (\%) & $6(13.0)$ & $7(16.7)$ & 0.4 \\
\hline Hydroxychloroquine, n (\%) & $38(82.6)$ & $32(66.7)$ & 0.08 \\
\hline Glucocorticoid, n (\%) & $42(91.3)$ & $42(87.5)$ & 0.7 \\
\hline Dosage, mg/day & $34.8 \pm 38.4$ & $13.9 \pm 11.7$ & $<0.001$ \\
\hline Low-dose ${ }^{\mathrm{a}}, \mathrm{n}(\%)$ & $22(47.8)$ & $34(70.8)$ & 0.01 \\
\hline High-dose $^{\mathrm{b}}, \mathrm{n}(\%)$ & $20(43.5)$ & $8(16.7)$ & 0.004 \\
\hline
\end{tabular}

Data are presented as the mean \pm standard deviation unless otherwise stated. ${ }^{a}<30 \mathrm{mg}$ prednisone or equivalent per day; ${ }^{b} \geq 30 \mathrm{mg}$ prednisone or equivalent per day. SLE, systemic lupus erythematosus; HZ, herpes zoster; dsDNA, double stranded DNA; ESR, erythrocyte sedimentation rate; CRP, C-reactive protein; SLEDAI, SLE Disease Activity Index; ANA, antinuclear antibodies; ACL, anticardiolipin antibody; Sm, Smith.

In total, 42 of the 46 patients $(91.3 \%)$ were undergoing GC treatment at the time of $\mathrm{HZ}$ onset, at a mean equivalent dose of $34.8 \pm 38.4 \mathrm{mg}$ (range, 5-200 $\mathrm{mg}$ ) prednisone. A total of 20 patients $(43.5 \%)$ received high-dose GC therapy, and the remaining 22 patients received low-dose GC therapy. Additional ISA were also administrated in 40 patients prior to the onset of HZ, including 20 with cyclophosphamide (CYC), 8 with mycophenolate mofetil (MMF), 6 with methotrexate (MTX) and 38 with hydroxychloroquine (HCQ).

A total of 5 patients $(10.9 \%)$ continued treatment with a previous dose of $\mathrm{GC}$ and the remaining 37 patients were treated with a decreased dose of GC (5.0-15.0 mg/day). Following the onset of HZ, HCQ was continuously prescribed in 33 patients and discontinued in the remaining 5 patients. CYC and MMF were discontinued in all patients throughout their $\mathrm{HZ}$ episodes. MTX treatment was discontinued in 4 patients and continued in the remaining 2 patients.

Comparison of study variables between SLE patients with and without HZ. Demographic data, clinical characteristics and administered treatments were compared between SLE patients with and without HZ (Table I). SLE patients with HZ presented with a significantly higher SLEDAI score $(9.1 \pm 7.4$ vs. $5.4 \pm 4.2 ; \mathrm{P}=0.004)$, a significantly higher proportion of active 
lupus (54.3 vs. 29.2\%; $\mathrm{P}=0.02)$, a significantly higher frequency of lymphopenia ( 37.0 vs. $12.5 \%$; $\mathrm{P}=0.008)$, and a significantly higher percentage of renal involvement (78.3 vs. $56.2 \% ; \mathrm{P}=0.03$ ) compared with SLE patients without HZ. Focusing on immunosuppressive treatment administered prior to the onset of $\mathrm{HZ}$ infection, the mean oral GC dose was significantly higher in SLE patients with $\mathrm{HZ}$ compared with non-HZ controls $(34.8 \pm 38.4$ vs. $13.9 \pm 11.7 \mathrm{mg} /$ day; $\mathrm{P}=<0.001)$. Instances of high-dose $\mathrm{GC}$ therapy prescription was significantly increased in $\mathrm{HZ}$ patients compared with non-HZ patients (43.5 vs. $16.7 \%$; $\mathrm{P}=0.004)$. The frequency of patients who received ISA therapy was not significantly different between the two groups.

Factors associated with the occurrence of HZ in SLE patients. The risk of developing HZ was calculated for patients with SLE with various predisposing factors. All variables with a significant association with HZ in univariate analysis (Table II) were further included in a stepwise multivariate logistic regression analysis. The following variables were found to be risk factors for the development of $\mathrm{HZ}$ in patients with SLE in multiple analysis: Lymphopenia $(\mathrm{OR}=4.6 ; 95 \% \mathrm{CI}=1.5-13.8 ; \mathrm{P}=0.006)$ and high-dose GC therapy $(\mathrm{OR}=4.3 ; 95 \% \mathrm{CI}=1.6-11.7$; $\mathrm{P}=0.004)$. Therefore, these factors may act as predictors for the occurrence of $\mathrm{HZ}$ in patients with SLE.

Comparison of study variables between $\mathrm{HZ}$ patients with and without complications. Of the 46 SLE patients with HZ, 11 developed complications and 35 did not. The comparison of the study variables between these two groups is presented in Table III. Patients with complications of HZ exhibited significantly higher SLEDAI scores than patients without complications $(13.0 \pm 8.5$ vs. $7.9 \pm 6.8 ; \mathrm{P}=0.04)$, which indicated that the disease was more active in patients with complications. Compared with patients without complications, patients with complications of $\mathrm{HZ}$ demonstrated a significantly higher percentage of lymphopenia (63.6 vs. $28.6 \%$; $\mathrm{P}=0.04$ ) and more abnormal hematologic findings (100 vs. $28.6 \%$; $\mathrm{P}<0.001)$. The mean oral prednisone dose and the percentage of patients who received ISA therapy were similar between these two groups.

Factors associated with complications of HZ in SLE patients. Variables associated with the onset of HZ complications, i.e., lymphopenia and SLEDAI scores, were included in the multivariate logistic regression model. Lymphopenia was the only predictor of complicated $\mathrm{HZ}$ in the final model with an OR of 15.2 (95\% CI=2.7-85.1; $\mathrm{P}=0.002)$. This finding indicated that SLE patients with lymphopenia were 15.2 times more likely to develop complications of $\mathrm{HZ}$ when compared with patients with normal lymphocyte count.

\section{Discussion}

VZV typically remains latent in cranial or spinal ganglia following resolution of a systemic infection (1). Reactivation, which tends to occur in elderly persons and immune compromised patients, induces a vesicular skin eruption accompanied by pruritus and dysesthesias (19). Minimal information is available in the literature describing the characteristics of $\mathrm{HZ}$ infection. To the best of our knowledge, the present study is the
Table II. Significant variables associated with occurrence of herpes zoster on univariate logistic regression.

\begin{tabular}{lcc}
\hline Variable & Crude OR (95\%CI) & P-value \\
\hline Lymphopenia & $4.1(1.4-11.7)$ & 0.008 \\
Renal involvement & $2.8(1.1-6.9)$ & 0.03 \\
Active lupus $^{\mathrm{a}}$ & $2.9(1.2-6.8)$ & 0.01 \\
High-dose GC $^{\mathrm{b}}$ & $3.8(1.5-10.0)$ & 0.006 \\
\hline
\end{tabular}

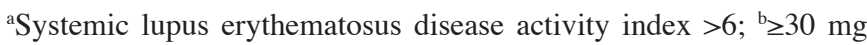
prednisone or equivalent per day. OR, odds ration; CI, confidence interval; GC, glucocorticoid.

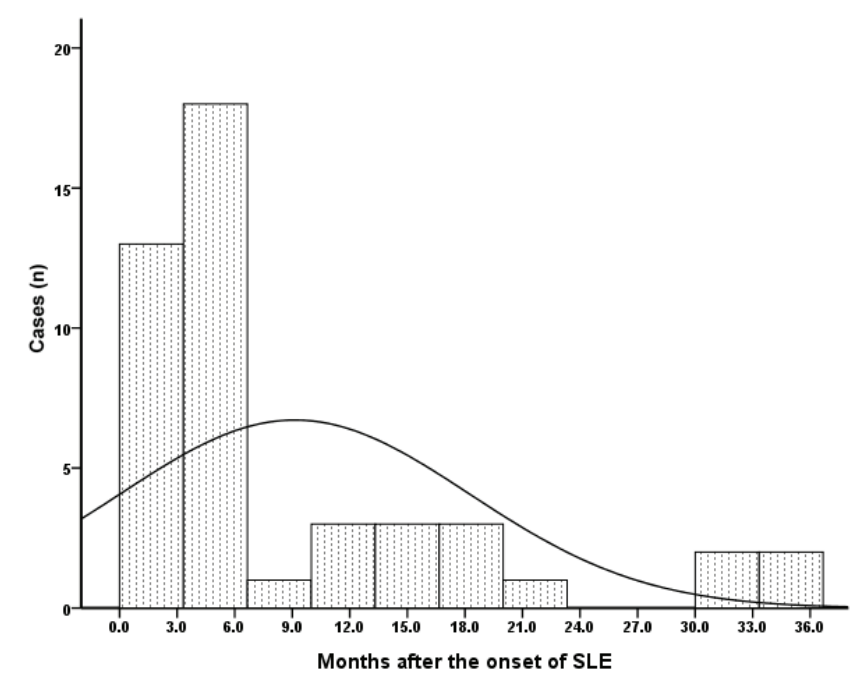

Figure 1. Time distribution of occurrence of $\mathrm{HZ}$ in relation to the onset of SLE. In the majority of patients $(67.4 \% ; 31 / 46)$, HZ was diagnosed within 3-6 months following SLE diagnosis, and rates reduced thereafter. HZ, herpes zoster; SLE, systemic lupus erythematosus.

first from Southern China to focus on SLE patients with HZ infection. Of note was the lower prevalence than that reported from other Asian regions. In a previous study from Saudi Arabia by Sayeeda et al (7), a total of 32 cases of $\mathrm{HZ}$ infection were identified among 624 SLE patients with a prevalence of $5.1 \%$. Ishikawa et al (6) previously reported that Japanese patients with SLE were vulnerable to $\mathrm{HZ}$ with an incidence of $46.6 \%$. There are two possible explanations for the lower prevalence in the present cohort. Firstly, the retrospective nature of the present study may have led to an underestimation of the incidence caused by mild HZ cases. Secondly, it may be due to the large number of patients who received HCQ, which had a wide range of antimicrobial effects. There are two previous studies that suggested that antimalarials may be protective against infections in patients with SLE $(20,21)$.

The present study also indicated that most instances of $\mathrm{HZ}$ occurred early in the course of the disease, with the peak occurrence of HZ at 3-6 months following SLE diagnosis. This time period was earlier than other reports. Nagasawa et al (22) previously summarized that almost half of the Japanese adult patients developed $\mathrm{HZ}$ in the first year following SLE diagnosis. Borba et al (4) observed that HZ was typically a 
Table III. Comparison of SLE patients with complicated and uncomplicated HZ.

\begin{tabular}{|c|c|c|c|}
\hline Characteristic & Complicated HZ $(n=11)$ & Uncomplicated HZ (n=35) & P-value \\
\hline \multicolumn{4}{|l|}{ Demographic characteristics } \\
\hline Sex, male:female & $0: 11$ & $2: 32$ & 1.0 \\
\hline Age, years & $32.1 \pm 13.4$ & $35.8 \pm 14.5$ & 0.5 \\
\hline Duration of SLE, years & $3.4 \pm 5.1$ & $2.9 \pm 4.4$ & 0.8 \\
\hline \multicolumn{4}{|l|}{ Laboratory data } \\
\hline ANA positive, n (\%) & $11(100)$ & $34(97.14)$ & 1.0 \\
\hline Anti-dsDNA positive, n (\%) & $7(63.6)$ & $20(57.1)$ & 0.7 \\
\hline Anti-Sm antibody positive, n (\%) & $1(9.1)$ & $2(5.7)$ & 0.7 \\
\hline $\mathrm{ACL}, \mathrm{n}(\%)$ & $1(9.1)$ & $2(5.7)$ & 0.7 \\
\hline Leucopenia, n (\%) & $2(18.2)$ & $5(14.3)$ & 0.8 \\
\hline Neutropenia, n (\%) & $3(27.3)$ & $3(8.6)$ & 0.1 \\
\hline Lymphopenia, n (\%) & $7(63.6)$ & $10(28.6)$ & 0.04 \\
\hline Monocytosis, n (\%) & $7(63.6)$ & $11(31.4)$ & 0.06 \\
\hline Anemia, n $(\%)$ & $8(72.7)$ & $21(60.0)$ & 0.5 \\
\hline Hypoproteinemia, n (\%) & $8(72.7)$ & $18(51.4)$ & 0.2 \\
\hline $\mathrm{ESR}, \mathrm{mm} / \mathrm{h}$ & $32.5 \pm 25.8$ & $38.4 \pm 32.5$ & 0.6 \\
\hline $\mathrm{CRP}, \mathrm{mg} / \mathrm{l}$ & $13.9 \pm 23.0$ & $28.3 \pm 71.0$ & 0.4 \\
\hline \multicolumn{4}{|l|}{ Clinical features } \\
\hline Renal involvement, n (\%) & $8(72.7)$ & $28(80.0)$ & 0.7 \\
\hline Neuro-psychiatric manifestations, n (\%) & $2(18.2)$ & $1(2.9)$ & 0.1 \\
\hline Hematological involvement, n (\%) & $11(100)$ & $10(28.6)$ & $<0.001$ \\
\hline Articular, n (\%) & $7(63.6)$ & $13(37.1)$ & 0.2 \\
\hline Mucocutaneous involvement, n (\%) & $7(63.6)$ & $14(40.0)$ & 0.2 \\
\hline Serositis, $\mathrm{n}(\%)$ & $1(9.1)$ & $4(11.4)$ & 1.0 \\
\hline SLEDAI & $13.0 \pm 8.5$ & $7.9 \pm 6.8$ & 0.04 \\
\hline Lupus active, n (\%) & $8(72.7)$ & $17(48.6)$ & 0.2 \\
\hline \multicolumn{4}{|l|}{ Treatments prior to $\mathrm{HZ}$ onset } \\
\hline Methotrexate, n (\%) & $2(18.2)$ & $4(11.4)$ & 0.6 \\
\hline Cyclophosphamide, n (\%) & $6(54.5)$ & $14(40.0)$ & 0.5 \\
\hline Mycophenolate motifile, n (\%) & $3(27.3)$ & $5(13.5)$ & 0.4 \\
\hline Hydroxychloroquine, n (\%) & $8(72.7)$ & $30(85.7)$ & 0.4 \\
\hline Glucocorticoid, n (\%) & $10(90.9)$ & $32(91.4)$ & 1.0 \\
\hline Dosage, mg/day & $43.9 \pm 28.9$ & $46.7 \pm 44.3$ & 0.7 \\
\hline High-dose $^{\mathrm{a}}, \mathrm{n}(\%)$ & $5(45.5)$ & $15(42.9)$ & 1.0 \\
\hline
\end{tabular}

${ }^{a} \geq 30 \mathrm{mg}$ prednisone or equivalent per day. SLE, systemic lupus erythematosus; HZ, herpes zoster; dsDNA, double stranded DNA; ESR, erythrocyte sedimentation rate; CRP, C-reactive protein; SLEDAI, SLE Disease Activity Index; ANA, antinuclear antibodies; ACL, anticardiolipin antibody; Sm, Smith.

late-SLE complication, as $>50 \%$ of $\mathrm{HZ}$ events occurred over 5 years following SLE diagnosis and only $7.9 \%$ within the first 2 years. In the present study, 2/3 of HZ was developed within 6 months after SLE diagnosis, which may be associated with activity of lupus or drugs. Therefore, the present findings suggested that clinicians must pay close attention to the latent vesicular skin eruption, particularly within the initial 3-6 months following SLE diagnosis.

Another notable finding was the higher prevalence of complications of $\mathrm{HZ}$ among the present cohort than that based on general population. The most common complication was postherpetic neuralgia, followed by superimposed bacterial infection. In the general population infected with HZ, postherpetic neuralgia occurs in $5-9 \%$ of people and is significantly more common in persons over age $60(23,24)$. In the present study, the rate of postherpetic neuralgia was in accordance with previous studies in SLE patients $(4,25)$, which was more frequent than that in the general population. Conversely, superimposed bacterial skin infection was observed in $8.7 \%$ of the present cohort, which was higher than the $1.4 \%$ reported in a previous general population-based study (3). All HZ patients in the present study exhibited full recovery and none of them experienced severe complications or succumbed to mortality. Therefore, despite a higher frequency of complications, 
complications with HZ infections in SLE patients were relatively benign.

Only a small number of studies have explored predictors for developing HZ in patients with SLE $(9,14,15)$, which have yielded inconsistent results. Furthermore, previous studies have rarely investigated the association between lymphopenia and HZ. The present study demonstrated that lymphopenia led to a risk of $\mathrm{HZ}$ infection and serious complications concomitant with HZ in patients with SLE. This finding was in accordance with two previous reports. $\mathrm{Ng}$ et al (26) demonstrated that HZ was more likely to develop in SLE patients with lymphopenia, and $\mathrm{Hu}$ et al (15) also suggested that in patients with SLE who developed HZ, the frequency of lymphopenia was increased compared with those without HZ. Although the exact mechanisms underlying the association between lymphopenia and VZV reactivation remain to be elucidated, it was hypothesized that decreased cell-mediated immunity serves a key role, as lymphopenia was a more specific reflection of defective cell-mediated immunity (CMI). The main defense against VZV reactivation in general populations was $\mathrm{CMI}$ rather than humoral immunity, as recurrent VZV infections occurred in patients with antibodies against VZV. A number of previous studies have demonstrated that CMI in patients with SLE may increase the risk of HZ. Nagasawa et al (22) suggested that the high incidence of $\mathrm{HZ}$ in patients with SLE was likely due to defects in CMI. Park et al (27) revealed that patients with lupus exhibited significantly lower VZV-specific cluster of differentiation $\mathrm{T}$ cell frequencies than rheumatoid arthritis patients and healthy controls. VZV-specific T cells have an important role in maintaining the equilibrium between the host and the virus during latency. A decline in the frequency of VZV-specific $\mathrm{T}$ cells has been demonstrated to be associated with an increased risk of VZV reactivation, leading to HZ (28). Furthermore, VZV itself has been reported to induce lymphopenia once reactivated, which may also have contributed to poor patient outcomes such as cutaneous dissemination, prolonged atypical skin lesions, ocular complications, and CNS involvement (29).

The present study demonstrated that high-dose GC ( $\geq 30 \mathrm{mg}$ prednisone or equivalent per day) was an independent risk factor for infection with $\mathrm{HZ}$ in patients with SLE. Similar results were previously reported by $\mathrm{Wu}$ et al (14) and Manzi et al (25), who noted that steroid usage increased the risk of $\mathrm{HZ}$ in a dose-dependent manner. Consistent with findings from Wu et al (14) and Sayeeda et al (7), the present study also demonstrated that treatment with additional ISA, including CYC, MMF and MTX, did not appear to confer higher risk. The present results highlighted the relevance of $\mathrm{GC}$ as the most important immunosuppressive drug in terms of risk of HZ infections in SLE. This suggests that careful monitoring of $\mathrm{HZ}$ occurrence is warranted in patients with SLE taking high-dose GC, and that discontinuing ISA therapy may be unnecessary during instances of $\mathrm{HZ}$ in patients with SLE. A prospective study with a larger sample size may be required to confirm the validity of this therapeutic strategy by following up HZ-infected patients.

In conclusion, this is, to the best of our knowledge the first cohort in Southern Chinese patients with SLE to determine that $\mathrm{HZ}$ has features including a relatively low prevalence and more common complications with a relatively benign course. In addition, HZ was demonstrated to be an early complication of SLE, with the highest risk of $\mathrm{HZ}$ within 3-6 months following SLE diagnosis. The present data supported the role of lymphopenia and high-dose GC therapy as risk factors for the occurrence HZ. Furthermore, lymphopenia was considered as an independent risk factor for severe $\mathrm{HZ}$ in patients with SLE. Therefore, these findings suggest that careful monitoring of $\mathrm{HZ}$ occurrence was warranted in SLE patients with risk factors. In addition, those with lymphopenia may benefit most from vaccination for $\mathrm{HZ}$.

\section{Acknowledgements}

The present study was supported by grants from the Guangdong Technology Project (grant nos. 2014A020221009, and 2016A020215043) and a grant of National Natural Science Foundation of China (grant no. 81603435).

\section{References}

1. Mahalingam R, Wellish M, Wolf W, Dueland AN, Cohrs R, Vafai A and Gilden D: Latent varicella-zoster viral DNA in human trigeminal and thoracic ganglia. N Engl J Med 323: 627-631, 1990.

2. Pope JE, Krizova A, Ouimet JM, Goodwin JL and Lankin M: Close association of herpes zoster reactivation and systemic lupus erythematosus (SLE) diagnosis: Case-control study of patients with SLE or noninflammatory nusculoskeletal disorders. J Rheumatol 31: 274-279, 2004.

3. Yawn BP, Saddier P, Wollan PC, St Sauver JL, Kurland MJ and Sy LS: A population-based study of the incidence and complication rates of herpes zoster before zoster vaccine introduction. Mayo Clin Proc 82: 1341-1349, 2007.

4. Borba EF, Ribeiro AC, Martin P, Costa LP, Guedes LK and Bonfá E: Incidence, risk factors, and outcome of Herpes zoster in systemic lupus erythematosus. J Clin Rheumatol 16: 119-122, 2010.

5. Chakravarty EF, Michaud K, Katz R and Wolfe F: Increased incidence of herpes zoster among patients with systemic lupus erythematosus. Lupus 22: 238-244, 2013.

6. Ishikawa O, Abe M and Miyachi Y: Herpes zoster in Japanese patients with systemic lupus erythematosus. Clin Exp Dermatol 24: 327-328, 1999.

7. Sayeeda A, Al Arfaj H, Khalil N and Al Arfaj AS: Herpes Zoster infections in SLE in a university hospital in Saudi Arabia: Risk factors and outcomes. Autoimmune Dis 2010: 174891, 2010.

8. Oomatia A, Fang H, Petri M and Birnbaum J: Peripheral neuropathies in systemic lupus erythematosus (SLE): Clinical features, disease associations, and immunological characteristics evaluated over a 25-year study period. Arthritis Rheumatol 66: 1000-1009, 2014.

9. Lee PP, Lee TL, Ho MH, Wong WH and Lau YL: Herpes zoster in juvenile-onset systemic lupus erythematosus: Incidence, clinical characteristics and risk factors. Pediatr Infect Dis J 25: 728-732, 2006.

10. Guthridge JM, Cogman A, Merrill JT, Macwana S, Bean KM, Powe T, Roberts V, James JA and Chakravarty EF: Herpes zoster vaccination in SLE: A pilot study of immunogenicity. J Rheumatol 40: 1875-1880, 2013.

11. Kimberlin DW and Whitley RJ: Varicella-zoster vaccine for the prevention of herpes zoster. N Engl J Med 356: 1338-1343, 2007.

12. Singh JA, Furst DE, Bharat A, Curtis JR, Kavanaugh AF, Kremer JM, Moreland LW, O'Dell J, Winthrop KL, Beukelman T, et al: 2012 update of the 2008 American College of Rheumatology recommendations for the use of disease-modifying antirheumatic drugs and biologic agents in the treatment of rheumatoid arthritis. Arthritis Care Res (Hoboken) 64: 625-639, 2012.

13. Papadopoulou D and Sipsas NV: Comparison of national clinical practice guidelines and recommendations on vaccination of adult patients with autoimmune rheumatic diseases. Rheumatol Int 34: 151-163, 2014.

14. Wu SA, Yeh KW, Yao TC and Huang JL: Association of herpes zoster infection with clinical characteristics and MBL2 gene polymorphisms in Chinese children with systemic lupus erythematosus. Pediatr Infect Dis J 30: 656-660, 2011. 
15. Hu SC, Lin CL, Lu YW, Chen GS, Yu HS, Wu CS and Lan CC: Lymphopaenia, anti-Ro/anti-RNP autoantibodies, renal involvement and cyclophosphamide use correlate with increased risk of herpes zoster in patients with systemic lupus erythematosus. Acta Derm Venereol 93: 314-318, 2013.

16. Eilertsen GØ, Becker-Merok A and Nossent JC: The influence of the 1997 updated classification criteria for systemic lupus erythematosus: Epidemiology, disease presentation, and patient management. J Rheumatol 36: 552-559, 2009.

17. Cohen JI: Clinical practice: Herpes zoster. N Engl J Med 369 255-263, 2013.

18. Bombardier C, Gladman DD, Urowitz MB, Caron D and Chang $\mathrm{CH}$ : Derivation of the SLEDAI. A disease activity index for lupus patients. The committee on prognosis studies in SLE. Arthritis Rheum 35: 630-640, 1992.

19. Brisson M, Edmunds WJ, Law B, Gay NJ, Walld R, Brownell M, Roos LL and De Serres G: Epidemiology of varicella zoster virus infection in Canada and the United Kingdom. Epidemiol Infect 127: 305-314, 2001

20. Sisó A, Ramos-Casals M, Bové A, Brito-Zerón P, Soria N, Muñoz S, Testi A, Plaza J, Sentís J and Coca A: Previous antimalarial therapy in patients diagnosed with lupus nephritis: Influence on outcomes and survival. Lupus 17: 281-288, 2008.

21. Ruiz-Irastorza G, Olivares N, Ruiz-Arruza I, Martinez-Berriotxoa A, Egurbide MV and Aguirre C: Predictors of major infections in systemic lupus erythematosus. Arthritis Res Ther 11: R109, 2009.

22. Nagasawa K, Yamauchi Y, Tada Y, Kusaba T, Niho Y and Yoshikawa H: High incidence of herpes zoster in patients with systemic lupus erythematosus: An immunological analysis. Ann Rheum Dis 49: 630-633, 1990.
23. Straus SE, Ostrove JM, Inchauspé G, Felser JM, Freifeld A, Croen KD and Sawyer MH: NIH conference. Varicella-zoster virus infections. Biology, natural history, treatment, and prevention. Ann Intern Med 108: 221-237, 1988.

24. Carroll S, Gater A, Abetz-Webb L, Smith F, Demuth D and Mannan A: Challenges in quantifying the patient-reported burden of herpes zoster and post-herpetic neuralgia in the UK: Learnings from the Zoster Quality of Life (ZQOL) study. BMC Res Notes 6: 486, 2013.

25. Manzi S, Kuller LH, Kutzer J, Pazin GJ, Sinacore J, Medsger TA Jr and Ramsey-Goldman R: Herpes zoster in systemic lupus erythematosus. J Rheumatol 22: 1254-1258, 1995.

26. Ng WL, Chu CM, Wu AK, Cheng VC and Yuen KY: Lymphopenia at presentation is associated with increased risk of infections in patients with systemic lupus erythematosus. QJM 99: 37-47, 2006.

27. Park HB, Kim KC, Park JH, Kang TY, Lee HS, Kim TH, Jun JB, Bae SC, Yoo DH, Craft J and Jung S: Association of reduced CD4 T cell responses specific to varicella zoster virus with high incidence of herpes zoster in patients with systemic lupus erythematosus. J Rheumatol 31: 2151-2155, 2004.

28. Cohen EJ: Prevention of herpes zoster: We need to do better. JAMA Ophthalmol 131: 396-398, 2013.

29. Levin MJ, Smith JG, Kaufhold RM, Barber D, Hayward AR, Chan CY, Chan IS, Li DJ, Wang W, Keller PM, et al: Decline in varicella-zoster virus (VZV)-specific cell-mediated immunity with increasing age and boosting with a high-dose VZV vaccine. J Infect Dis 188: 1336-1344, 2003. 